\title{
Problems of Using Hipparcos Astrometry of Double Stars
}

\author{
S.E. Urban, T.E. Corbin, G.L. Wycoff, and B.D. Mason \\ U.S. Naval Observatory, Washington DC
}

\begin{abstract}
The current optical realization of the International Celestial Reference System is the Hipparcos main catalog in its entirety. This paper demonstrates that a subset of the main catalog stars - specifically those marked as double ("C" stars), acceleration solution ("G"), variability induced movers ("V"), and stochastic solution stars ("X") - is of much lower quality than the majority of the catalog. Stars marked as suspected doubles ("S") were investigated and found to show few problems. The authors have put forth a resolution (Resolution 2, this conference) to remove the $\mathrm{C}, \mathrm{G}, \mathrm{V}$, and $\mathrm{X}$ stars, in addition to the Hipparcos orbit stars ("O stars"), from the optical realization of the reference frame.
\end{abstract}

\section{Introduction}

IAU resolution B2 of the XXIII General Assembly (IAU 1998) specifies that the Hipparcos Catalog (ESA, 1997) is the optical realization of the ICRS. However, this includes a class of stars - those in multiple systems - having a significantly lower quality of proper motions on average than the majority of stars in Hipparcos.

In the following sections, the internal standard errors of the Hipparcos Catalog are utilized to show a clear difference between those stars believed to be single and those that are not. Additionally, comparisons utilizing the recently released Tycho-2 Catalogue (Høg et al., 2000) show that the standard errors for the double stars may be underestimated by as much as $30 \%$.

With these results, the authors propose to amend resolution B2 to eliminate known multiple systems and problem stars from the optical realization of the ICRS. The numbers of stars in each category are:

\begin{tabular}{|l|l|r|}
\hline type & H59 code (MultFlag) & number of stars \\
\hline All stars w/ astrometry & all & 117,955 \\
Suspected doubles & S (H61) & 7,624 \\
Double & C & 13,211 \\
Problem & G,V,X,O & 4706 \\
\hline
\end{tabular}

\section{Data Utilized}

In the following comparisons, those stars identified in both the Hipparcos main catalog and Tycho-2 catalog were used. Note that stars only appearing in the 
Hipparcos annexes were excluded, as they are not covered by the IAU resolution. Additionally, stars in Tycho-2 whose proper motions were from photocenter data (blended images) were rejected. No stars identified as orbit stars in Hipparcos were included. The following table gives the number of stars used in the following comparisons. Note that Hipparcos observed a significantly higher number of double stars than are used in these comparisons. The greater resolution of the Hipparcos instrument over that of Tycho and the ground-based data used in the Tycho-2 proper motions resulted in many close doubles being photocenters in the Tycho-2 catalog. These are not included in the comparisons.

\begin{tabular}{|l|l|r|c|}
\hline type & H59 code (MultFlag) & number of stars & $\left\langle\bar{B}_{T}\right\rangle$ \\
\hline Single stars & no code & 92,316 stars & $9.2 \mathrm{mag}$ \\
Suspected doubles & S (H61), non-X & 6556 stars & $9.9 \mathrm{mag}$ \\
Double stars & $\mathrm{C}$ & 6707 stars & $9.3 \mathrm{mag}$ \\
Other problem stars & $\mathrm{G}, \mathrm{V}$, or X & 4177 stars & $9.4 \mathrm{mag}$ \\
\hline
\end{tabular}

\section{Comparisons}

Hipparcos Quoted Errors For the four groups of stars (single, suspected double, double, problem), histograms of the Hipparcos proper motion errors have been plotted in Figure 1. Note that these are taken directly from the Hipparcos catalog; no external data are utilized. Clearly, there is a marked difference in precision between the single and suspected double stars versus the non-single stars.

Comparisons of Hipparcos Proper Motions with Tycho-2 For the four groups of stars (single, suspected double, double, problem), histograms of the differences between the Hipparcos proper motions and those of Tycho- 2 have been plotted, as shown in Figure 2. Not surprisingly, the differences are much larger for the double and problem stars than for the single and suspected double stars.

For both the double and problem stars, significant portions of the data extend beyond the area covered by the graph. These amounts are displayed in the bars to the extreme right and left in the plots.

Comparisons of Hipparcos Proper Motion errors with those of Tycho-2 There is a clear difference between the internal errors quoted in Hipparcos for the single and the $\mathrm{S}$ stars versus the $\mathrm{C}, \mathrm{G}, \mathrm{V}$, and $\mathrm{X}$ stars. An evaluation of the under- or over-estimation of those errors is warranted. Utilizing a technique recently used by Hoogerwerf \& Blaauw (2000), we have compared the errors in proper motion of Hipparcos and Tycho-2 for the four groups.

To compare the errors, a normalized proper motion difference for each coordinate for each star in computed. This difference, $\Delta$, is defined as:

$$
\Delta=\frac{\mu_{H}-\mu_{T}}{\sqrt{\sigma_{\mu_{H}}^{2}+\sigma_{\mu_{T}}^{2}}}
$$

where $\mu_{H}$ and $\mu_{T}$ are the proper motions in Hipparcos and Tycho-2, and $\sigma_{\mu_{H}}$ and $\sigma_{\mu_{T}}$ are the errors in proper motion quoted in the two catalogs. 

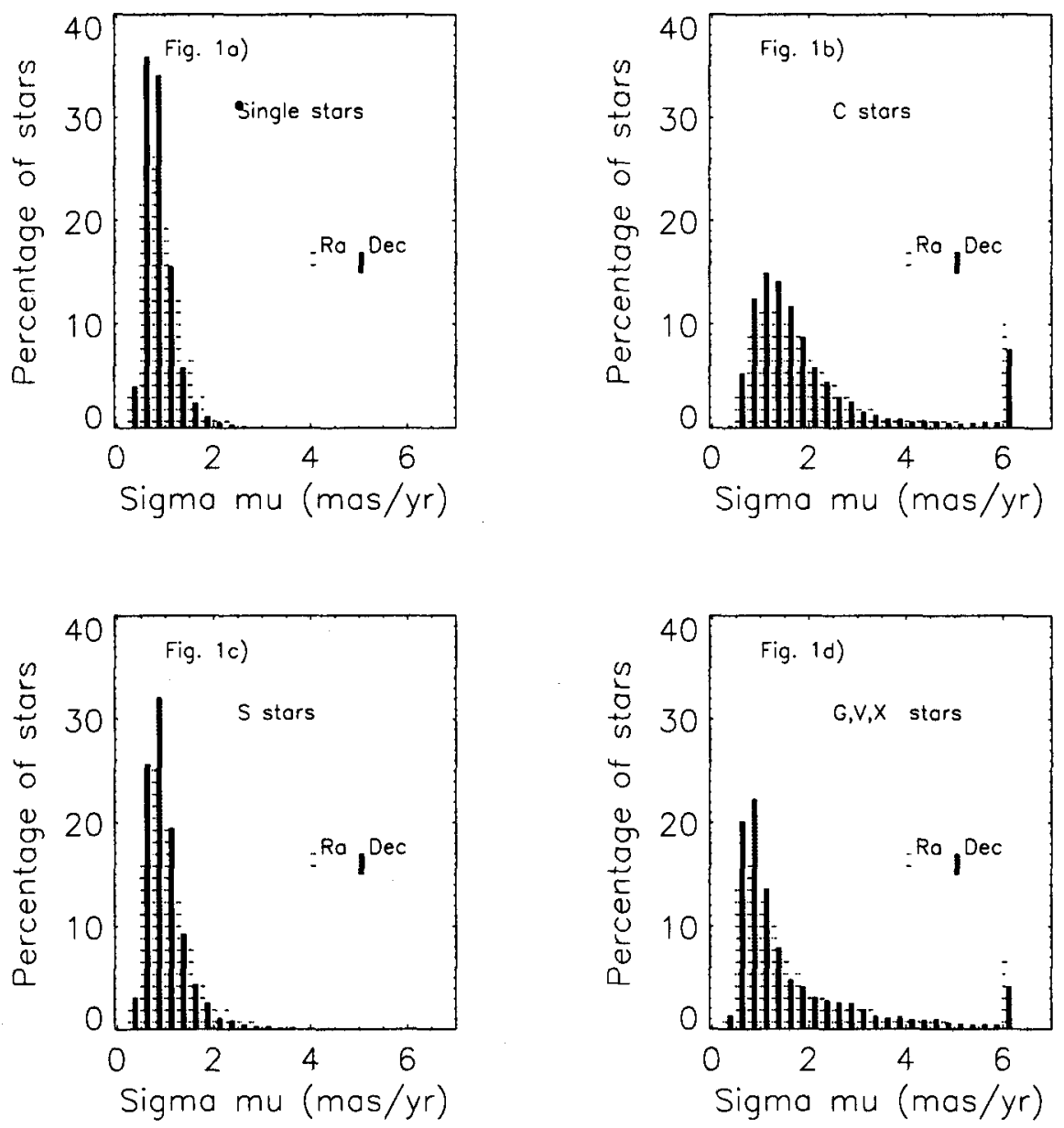

Figure 1. Percentage of stars by quoted Hipparcos standard error. 

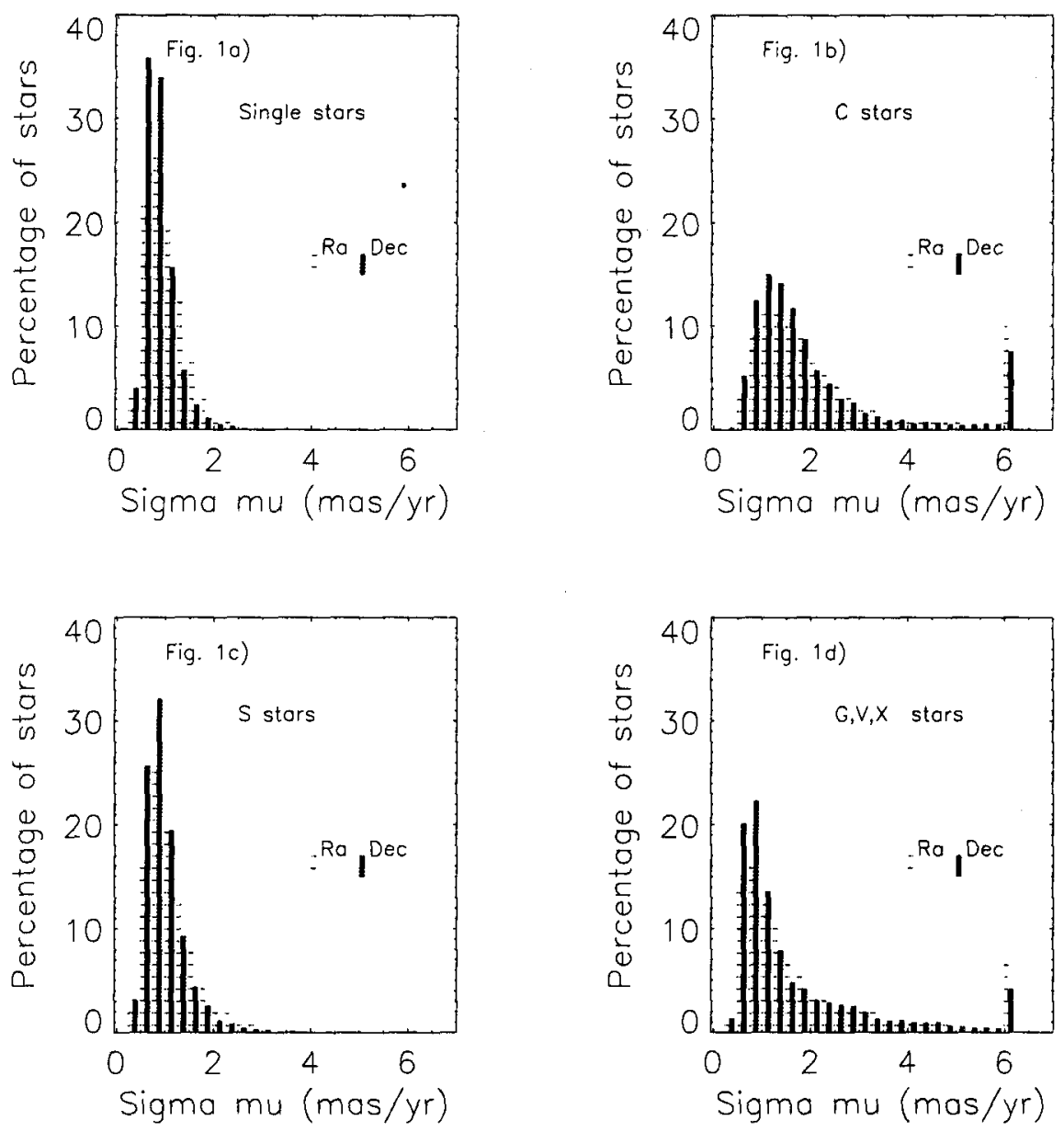

Figure 2. Difference between Hipparcos and Tycho-2 proper motions. Note the large wings on the double and G, V, and X stars. 
Define $F_{H}\left(\mu_{H}\right)$ which gives the probability of observing proper motion $\mu_{H}$ when the real proper motion is $\hat{\mu}_{H}$, and the real proper motion error is $\hat{\sigma}_{\mu_{H}}$. A similar function is defined for the Tycho-2 proper motions and errors.

Assuming that the two catalogs are independent, the mean of $\Delta$ should be zero, because

$$
\bar{\Delta}=E(\Delta)=\frac{\hat{\mu}_{H}-\hat{\mu}_{T}}{\sqrt{\sigma_{\mu_{H}}^{2}+\sigma_{\mu_{T}}^{2}}},
$$

and $\hat{\mu}_{H}=\hat{\mu}_{T}$ since they are defined as the real proper motion of the same star. The variance of $\Delta$ is

$$
\sigma_{\Delta}^{2}=E\left([\Delta-E(\Delta)]^{2}\right)=\frac{\hat{\sigma}_{\mu_{H}}^{2}+\hat{\sigma}_{\mu_{T}}^{2}}{\sigma_{\mu_{H}}^{2}+\sigma_{\mu_{T}}^{2}} .
$$

If the quoted standard deviations are correct, then $\sigma_{\Delta}$, should be unity.

The value of $\sigma_{\Delta}$ can be below 1.0 if the catalogs are correlated or the errors in one or both catalogs are over-estimated. Similarly, $\sigma_{\Delta}$ will be above 1.0 if one or both of the catalog errors are under-estimated.

\begin{tabular}{|c|c|}
\hline$\sigma_{\Delta}$ & reasons \\
\hline Less than 1.0 & catalogs correlated \\
Less than 1.0 & quoted errors over-estimated \\
Greater than 1.0 & quoted errors under-estimated \\
\hline
\end{tabular}

\section{Discussion}

The following table shows the standard deviations of the normalized differences for each of the four groups of Hipparcos stars as compared with Tycho-2. A three sigma outlier removal was made.

\begin{tabular}{|l|l|}
\hline type & $\sigma_{\Delta}$ \\
\hline Single & 0.91 \\
Suspected double; S & 1.00 \\
Double; C & 1.54 \\
Problem; G, V, and X & 2.01 \\
\hline
\end{tabular}

The single stars have a standard deviation below one, most likely due to the two catalogs not being truly independent. (This correlation is a result of utilizing the Hipparcos data to reduce all of the catalogs used to compute the Tycho2 proper motions.) The suspected doubles have a standard deviation of one, indicating a non-significant underestimation of errors. Both the doubles and the G, V, and X group show that either Hipparcos, Tycho-2, or both catalogs' proper motion errors are underestimated for the non-single stars. The combined underestimation is significant; values of $50 \%$ for the $\mathrm{C}$ stars and $100 \%$ for the $\mathrm{G}, \mathrm{V}$, and $\mathrm{X}$ stars are indicated (and this does not account for the fact that the Tycho-2 and Hipparcos catalogs are correlated, which should lower the standard deviation). 
It is impossible to tell if only one of the catalogs is to blame. When selected double stars are used, as shown in the table below, the underestimation factor is still large. It is suspected that the contributions to the underestimation of the errors are probably not due to one class of star, nor are they from only one of the catalogs.

Some causes can be assumed. With better a priori information, Falin \& Mignard (1999), Söderhjelm (1999), and Fabricius \& Makarov (2000) have all recently re-analyzed some Hipparcos double stars, quite often changing the proper motions by over $100 \mathrm{mas} / \mathrm{yr}$. It is also known that for stars with orbital periods of a few decades, the 3.5-year observing span of Hipparcos is insufficient in duration to adequately separate proper motion from that of orbital motion. Additionally, astrometry from transit circles and photographic plates becomes very questionable for double stars with separations of a few arcseconds and smaller.

It is the authors' intention to continue this investigation to try to uncover some of the reasons for these results. However, in view of these results and those shown in Figure 1, there is no question that the representation of the optical reference frame is best made by avoiding the non-single stars.

Selected double stars The following table shows the standard deviations for the normalized differences of selected groups of Hipparcos double (C) stars. As stated earlier, if quoted errors in Hipparcos and Tycho-2 are correct, the standard deviation should be 1.0. The amount above 1.0 is the underestimation factor. These values are computed after a removal of outliers at the $3 \sigma$ level. The last line in the table gives the most selective group, that is stars that should have few problems in either Tycho-2 or Hipparcos. All stars have 5 or more positions going into the Tycho-2 motions, are in widely separated pairs, and have low quoted errors in both catalogs. Even so, underestimation in one or both of the catalogs amounts to nearly $30 \%$.

\begin{tabular}{|l|l|l|l|l|l|}
\hline $\begin{array}{l}\text { Catalog } \\
\text { positions } \\
\text { used in Tycho-2 }\end{array}$ & $\begin{array}{l}\text { Separation of } \\
\text { components }\end{array}$ & $\begin{array}{l}\text { limits on } \\
\text { errors } \\
\text { (mas/yr) }\end{array}$ & \# stars & $\sigma_{\Delta}$ & $\sigma_{\Delta}^{2}$ \\
\hline 5 or more & all & none & 4637 & 1.44 & 2.00 \\
5 or more & $>5.0$ & none & 1692 & 1.35 & 1.82 \\
5 or more & $>5.0$ & $2.5(\mathrm{H}) 3.5(\mathrm{~T})$ & 1195 & 1.27 & 1.62 \\
\hline
\end{tabular}

\section{References}

ESA, 1997, The Hipparcos and Tycho Catalogues, ESA SP-1200.

Fabricius, C. \& Makarov, V.V., 2000, Hipparcos astrometry for 257 stars using Tycho-2 data, Astron. Astrophys., submitted.

Falin, J.L. \& Mignard, F., 1999, Mining in the Hipparcos raw data, Astron. Astrophys. Sup., 135, 231.

Hartkopf, W., Mason, B., \& Worley, C., 2000, Fifth Catalog of Orbits of Visual Binary Stars, Pub. of the US Naval Observatory, in preparation. 
Høg, E., Fabricius, C., Makarov, V.V., Urban, S., Corbin, T., Wycoff, G., Bastian, U., Schwekendiek, P. \& Wicenec, A., 2000, The Tycho-2 Catalogue of the 2.5 Million Brightest Stars, Astron. Astrophys., 355, L27.

Hoogerwerf, R. \& Blaauw, A., 2000, The Hipparcos, Tycho, TRC \& ACT catalogues: A whole sky comparison of the proper motions, Astron. Astrophys., submitted.

IAU 1998, Trans. IAU, 23B.

Söderhjelm, S., 1999, Visual binary orbits Post-Hipparcos, Astron. Astrophys., $341,121$. 